\title{
Caracterização do microclima dos diferentes layouts de caixas no transporte de ovos férteis
}

\author{
Aérica C. Nazareno', Iran J. O. da Silva ${ }^{1}$, Frederico M. C. Vieira ${ }^{2}$, \\ Juliano R. de Camargo ${ }^{1}$ \& Sérgio R. R. de Medeiros ${ }^{1}$
}

\begin{abstract}
RESUMO
Quando as condições microclimáticas e de transporte são combinadas inadequadamente, os componentes dos ovos férteis podem ser danificados. Objetivou-se, com este trabalho, caracterizar e analisar a dependência espacial dos perfis microclimáticos no transporte de ovos férteis em diferentes layouts de caixas. A pesquisa foi conduzida na empresa integradora avícola, em Mogi Mirim, SP, por meio do acompanhamento de cinco carregamentos. Utilizou-se um caminhão climatizado do tipo baú, com capacidade para 592 caixas de ovos e se avaliaram dois layouts da carga (caixas superiores e inferiores) de 9 pilhas com seis caixas de ovos férteis. Um microprocessador foi instalado dentro das caixas de ovos, totalizando 18 microprocessadores. As variáveis meteorológicas analisadas foram: temperatura $\left({ }^{\circ} \mathrm{C}\right)$ umidade relativa $(\%)$ e entalpia específica ( $\mathrm{kJ} \mathrm{kg}^{-1}$ ar seco). O delineamento experimental adotado foi o inteiramente casualizado com parcelas subdividas e realizada a análise geoestatística (krigagem ordinária). No layout das caixas superiores foram registrados valores elevados de temperatura e baixos valores de umidade relativa e entalpia específica. As regiões de piores condições microclimáticas nos dois layouts se situaram nas partes central e traseira do caminhão, durante o transporte dos ovos férteis.
\end{abstract}

Palavras-chave: avicultura, ambiência, operações pré-porteira, logística

\section{Characterization of microclimate in different layout of boxes during transport of fertile eggs}

\begin{abstract}
The fertile egg components may be damaged when the microclimatic conditions and transportation characteristics are combined inadequately. Thus, the aim of this study was to characterize and to assess the spatial dependence of microclimatic profiles during transport of fertile eggs with different layout of boxes. A trial was conducted in a commercial poultry industry in Mogi Mirim in the State of São Paulo, Brazil, through monitoring five loads of hatching eggs. An air conditioned truck was used, with maximum capacity of 592 egg boxes. Two layout of boxes were assessed in the present study. One data logger was installed inside the egg boxes, totalizing 18 data loggers. The following thermal variables were studied: dry-bulb temperature $\left({ }^{\circ} \mathrm{C}\right)$, relative humidity $(\%)$ and specific enthalpy $\left(\mathrm{kJ} \mathrm{kg}^{-1} \mathrm{dry}\right.$ air). A completely randomized design was adopted, with a split-plot structure and was analysed through geostatistics (ordinary kriging). Inside the superior box layout, high values of temperature and lower values of relative humidity and specific enthalpy were registered. The worst regions for microclimatic in both layouts were located in the central and rear part of the truck during transport of fertile eggs.
\end{abstract}

Key words: poultry production, animal environment, prehatching operation, logistics

ESALQ/USP, Av. Pádua Dias 11, Agronomia, CEP 13418-900, Piracicaba, SP. Fone: (19) 3447-8563. E-mail: aericacn@yahoo.com.br; ijosilva@esalq.usp.br; juliano@flamboia.com.br, sergiogen@hotmail.com

2 UTFPR - Campus Dois Vizinhos, Estrada para Boa Esperança, km 04, C.P. 157, CEP 85660-000, Comunidade São Cristóvão Dois Vizinhos, PR. E-mail: fredvzoo@gmail.com 


\section{INTRODUÇÃO}

A ambiência pré-porteira consiste no conjunto de todas as operações envolvidas desde bisavozeiros até o transporte dos pintos de um dia, ou seja, todas as operações que antecedem a chegada do pinto de corte na granja, com destaque no transporte de ovos férteis entre o matrizeiro e o incubatório. $\mathrm{O}$ transporte de ovos ocorre frequentemente em diferentes condições climáticas e combinações de distâncias e horários. Essas combinações influenciam,em contrapartida, na qualidade do produto final (ovo fértil) e são, sem dúvida, as principais responsáveis pelas perdas nesta etapa (morte do embrião, máformação embrionária, baixa eclodibilidade e pintos de corte de baixa qualidade).

O impacto econômico das perdas decorrentes das operações pré-porteira em 2010 no Brasil foi alto, tomando-se como exemplo a produção de 28,851 bilhões de ovos que corresponde aos ovos férteis, incubados, e pelo consumo humano (ANUALPEC, 2011). Atualmente, o custo médio de produção por ovo fértil é em torno de 80 centavos, nos dez maiores estados produtores do Brasil; de acordo com esses dados, perdas de apenas $1 \%$ provocadas por transportes inadequados e/ou operações executadas de maneira errada na etapa pré-porteira podem ter gerado um prejuízo anual na avicultura de corte nacional, na ordem de 230 milhões de reais.

A combinação do microclima dentro do caminhão baú com a fisiologia do ovo é complexa em virtude de uma gama de condições climáticas encontradas em todo o Brasil observandose uma grande amplitude térmica ao longo do ano. A exposição do ovo fértil em temperatura e umidade relativa elevadas antes do armazenamento é prejudicial ao desenvolvimento embrionário (Yassin et al., 2008; Tanure et al., 2009). Por isto é importante o controle das condições do microclima do ambiente após a postura para evitar perdas produtivas nessas etapas.

Recomenda-se, para o transporte dos ovos férteis, que a temperatura ambiente do baú esteja abaixo do zero fisiológico $\left(20^{\circ} \mathrm{C}\right)$ com vista a garantir a paralisação do desenvolvimento embrionário antes que os ovos sejam colocados nas incubadoras (Fasenko et al., 1991; 1992; Elibol \& Brake, 2008a; 2008b). A umidade relativa ideal nesta etapa deve estar próxima de $75 \%$ evitando perda excessiva de água e a condensação na casca o que, provavelmente, propiciará um ambiente favorável para a proliferação microbiana (Araújo et al., 2009; Baracho et al., 2010).

Ao avaliar a distribuição térmica ao longo do caminhão de transporte de ovos comerciais, Damron et al. (1994) e Gast et al. (2000) observaram que os maiores desvios de temperatura estavam perto das portas e próximo ao climatizador. Outras pesquisas posteriores também demonstraram que as estações do ano influenciaram na temperatura interna do baú do caminhão, interferindo, sem dúvida, na temperatura interna dos ovos durante o transporte (Anderson et al., 2008). Barbosa Filho et al. (2009) observaram regiões críticas do ponto de vista microclimático nas partes central e traseira no transporte de frangos de corte adultos, tornando essas regiões inadequadasdo o ponto de vista de conforto térmico.

Desta forma, a problemática apresentada acima necessita de soluções no setor avícola, por meio da revisão dos processos de manejo, especialmente com relação às etapas pré-porteira. Devido à elevada quantidade de perdas e com base na necessidade de adequação deste manejo às condições ambientais de clima tropical objetivou-se, por meio desta pesquisa, caracterizar e analisar a dependência espacial dos perfis microclimáticos no transporte de ovos férteis em diferentes layouts de caixas.

\section{Material e Métodos}

A pesquisa foi conduzida nas instalações do matrizeiro e do incubatório avícola de uma empresa integradora localizada no município de Mogi Mirim, no estado de São Paulo, apresentando latitude de $22^{\circ} 25^{\prime} 55^{\prime \prime} \mathrm{S}$, longitude de $46^{\circ} 57^{\prime}$ $28^{\prime \prime} \mathrm{W}$ e altitude de 632 m;o período experimental foi de 1 mês durante o qual foram acompanhados cinco carregamentos durante o inverno.

Foi realizado um perfil térmico do caminhão de transporte tipo baú, modelo Constellation 26-370 $6 \times 4$ tractor da Volkswagen, apresentando as seguintes dimensões 7,5 x 2,6 x 2,6 m, com três eixos, a calibragem dos pneus com 100 libras, tipo de suspensão com molas parabólicas e amortecedores hidráulicos de dupla ação. $\mathrm{O}$ equipamento de climatização era regulado para as condições de temperatura de $23^{\circ} \mathrm{C}$ e umidade relativa de $80 \%$. A capacidade de carregamento do caminhão era de aproximadamente 592 caixas de ovos empilhadas e distribuídas na área da carroceria. Porém os carregamentos estudados apresentaram 444 caixas sendo formadas pilhas ao longo do caminhão com 6 caixas cada uma.

As caixas de transporte de ovos possuíam as dimensões externas de $63 \times 32,5 \times 30 \mathrm{~cm}$ e espessura $3 \mathrm{~mm}$ com aberturas nas laterais de $3,2 \times 1,1 \mathrm{~cm}$ e no fundo de $2 \times 2 \mathrm{~cm}$, as quais suportavam 240 ovos que equivalem a 8 bandejas de plástico com as dimensões de $30 \times 30 \mathrm{~cm}$ com capacidade de 30 ovos.

Foram realizados os monitoramentos de cinco viagens contendo o mesmo percurso $(60 \mathrm{~km})$, caminhão e motorista, durante os meses de agosto e setembro de 2010.

$\mathrm{Na}$ caracterização do ambiente térmico do baú climatizado foram registradas as variáveis: temperatura $\left(\mathrm{T},{ }^{\circ} \mathrm{C}\right)$ e umidade relativa (UR,\%) por meio de microprocessadores da marca Logen $\AA$, em intervalos de registro dos dados de 1 min.

Foram avaliados dois layouts da carga (caixas superiores e inferiores) de 9 pilhas de seis caixas de ovos férteis identificadas com etiquetas e distribuídas ao longo do perfil da carroceria do caminhão. Para obtenção do perfil das variáveis ambientais ao longo da carga do caminhão, foram instalados 9 microprocessadoresem toda a extensão do caminhão, das caixas superiores e inferiores das pilhas identificadas totalizando 18 microprocessadores, o que permitiu caracterizar o microclima cujos ovos férteis foram transportados (Figura 1).

Os valores de temperatura, umidade relativa e pressão atmosférica local foram utilizados para o cálculo da entalpia específica (h, $\mathrm{kJ} \mathrm{kg}^{-1}$ ar seco) por meio da Eq. 1, proposta por Rodrigues et al. (2011):

$$
\mathrm{h}=1,006 \mathrm{t}+\frac{\mathrm{UR}}{\mathrm{p}_{\mathrm{a}}} 10^{\frac{7,5 \mathrm{t}}{237,3+\mathrm{t}}}(71,28+0,052 \mathrm{t})
$$


A.

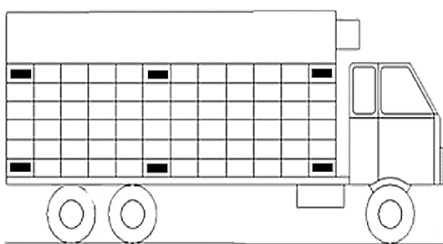

B.

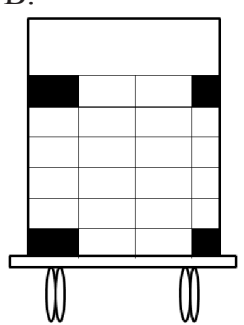

C.

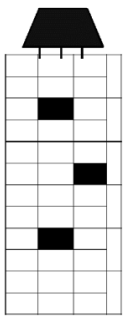

Figura 1. Distribuição dos microprocessadores representados pelas caixas pretas ao longo da carga vista da lateral (A), traseira (B) e superior do caminhão (C) de transporte de ovos férteis

em que:

h - entalpia específica, $\mathrm{kJ} \mathrm{kg}^{-1}$ ar seco

t $\quad$ - temperatura, ${ }^{\circ} \mathrm{C}$

$\mathrm{p}_{\mathrm{a}} \quad$ - pressão atmosférica, $\mathrm{mmHg}$

UR - umidade relativa, $\%$

O delineamento experimental foi o inteiramente casualizado (DIC) com parcelas subdividas, em que os layouts das cargas (caixas superiores e inferiores) foram alocados nas parcelas, os carregamentos nas subparcelas, com cinco repetições. Para comparação múltipla das médias utilizou-se o teste de Tukey a nível de $5 \%$ de probabilidade, por meio do software estatístico SAS (SAS, 2009).

Com a finalidade de caracterizar a dependência espacial das variáveis ambientais temperatura, umidade relativa e entalpia específica, utilizaram-se a geoestatística (krigagem ordinária) para obtenção dos demais pontos da carroceria do caminhão e a avaliação da dependência espacial das variáveis ambientais considerando-se como área de estudo o interior da carroceria do caminhão. Em cada conjunto de dados as amostras foram divididas em dois layouts das caixas de ovos superiores e inferiores, com o auxílio do programa $\mathrm{GS}^{+} 7.0$ (Robertson, 2000).

Para análise da geoestatística os dados foram submetidos à análise estatística descritiva e exploratória, conforme proposto por Libardi et al. (1996) para verificação da presença de pontos discrepantes (outliers). Na análise geoestatística esses valores provocam forte impacto, sobremaneira na parte inicial do semivariograma, fato que pode conduzir a conclusões erradas sobre a variância do ruído (efeito pepita) e/ou existência de correlação espacial.

$\mathrm{O}$ ajuste de modelos teóricos (esférico, exponencial e gaussiano) se deu através do cálculo do semivariograma, conforme a Eq. 2, proposta por Vieira (2000):

$$
\gamma^{\wedge}(\mathrm{h})=\frac{1}{2 \mathrm{~N}(\mathrm{~h})} \sum_{\mathrm{i}=1}^{\mathrm{N}(\mathrm{h})}[\mathrm{Z}(\mathrm{xi})-\mathrm{Z}(\mathrm{xi}+\mathrm{h})]^{2}
$$

em que:

$\mathrm{N}$ (h) - número de pares experimentais de observações

$\mathrm{Z}(\mathrm{xi})$ e $\mathrm{Z}(\mathrm{xi}+\mathrm{h})$ separados por uma distância $\mathrm{h}$

O semivariograma é representado pelo gráfico $\gamma^{\wedge}(\mathrm{h})$ versus h. Do ajuste de um modelo matemático aos valores calculados de $\gamma^{\wedge}(h)$ são estimados os coeficientes do modelo teórico para o semivariograma (o efeito pepita, $\mathrm{C}_{0}$; patamar, $\mathrm{C}_{0}+\mathrm{C}_{1}$ e o alcance, a).

Foram estimados os seguintes coeficientes: efeito pepita $\left(\mathrm{C}_{0}\right)$, patamar $\left(\mathrm{C}_{0}+\mathrm{C}_{1}\right)$, componente estrutural $\left(\mathrm{C}_{1}\right)$ e alcance de dependência espacial (a) para os semivariogramas. A escolha do melhor modelo baseou-se na menor soma do quadrado dos resíduos (SQR) e no coeficiente de determinação múltipla $\left(\mathrm{R}^{2}\right)$. Na sequência, também foi utilizado o $R^{2}$ da validação cruzada $\left(\mathrm{R}^{2}-\mathrm{VC}\right)$ (valores observados versus valores estimados). Para a análise do índice de dependência espacial (IDE) dos atributos utilizaram-se a relação definida no programa $\mathrm{GS}^{+}\left(\mathrm{C}_{1} / \mathrm{C}_{0}+\mathrm{C}_{1}\right)$ e os intervalos propostos por Zimback (2001) que considera a dependência espacial fraca (IDE $\leq 25 \%)$; moderada $(25 \%<$ IDE $<75 \%$ ) e forte (IDE $\geq 75 \%$ ).

Com o ajuste dos modelos teóricos e a definição dos coeficientes para os semivariogramas utilizou-se do método de estimativa de valores de atributos distribuídos no espaço a partir de valores adjacentes, conhecido como krigagem ordinária; trata-se de um método de estimativa por médias móveis. A krigagem é o método de interpolação da geoestatística que usa a dependência espacial expressa no semivariograma entre pontos amostrais vizinhos para estimar valores em qualquer posição dentro do campo, sem tendência e com variância mínima (Trangmar et al., 1985).

\section{Resultados e Discussão}

Foi notória a diferença $(\mathrm{P}<0,05)$ entre os layouts superiores e inferiores paratemperatura, umidade relativa e entalpia específica (Tabela 1); apesar disto, a diferença entre os layouts foi pequena para todas as variáveis o que sugere, sem dúvida, a falta do componente espacial, isto é, a variação dos fatores ao longo do caminhão pode aumentar a diferença entre os tratamentos.

Tabela 1. Valores médios e desvio padrão das variáveis temperatura $(T)$, umidade relativa (UR) e entalpia específica (h) em relação aos tratamentos de layouts das caixas superiores e inferiores no transporte dos ovos férteis

\begin{tabular}{lccc}
\hline \multicolumn{1}{c}{ Layouts } & $\begin{array}{c}\mathbf{T} \\
\left({ }^{\circ} \mathbf{C}\right)\end{array}$ & $\begin{array}{c}\text { UR } \\
\mathbf{( \% )}\end{array}$ & $\begin{array}{c}\mathbf{h} \\
\mathbf{( k J ~ k g}^{-1} \text { ar seco) }\end{array}$ \\
Caixas superiores & $23,6 \mathrm{a} \pm 1,6$ & $83 \mathrm{~b} \pm 13$ & $60,9 \mathrm{~b} \pm 3,6$ \\
Caixas inferiores & $23,0 \mathrm{~b} \pm 1,7$ & $84 \mathrm{a} \pm 14$ & $62,2 \mathrm{a} \pm 3,6$ \\
\hline CV \% & 5 & 11 & 5 \\
\hline Teste F & $217,51^{\star}$ & $289,30^{\star}$ & $83,95^{\star}$ \\
\hline
\end{tabular}

Na mesma coluna médias seguidas da mesma letra não diferem entre si a nível de 0,05 de probabilidade pelo teste de Tukey

* significativo a $0,05(P<0,05)$

Os maiores valores médios de temperatura, porém, foram encontrados no layout das caixas de ovos superiores com $23,6{ }^{\circ} \mathrm{C}$. Isto evidencia a aplicação do princípio da termodinâmica, em que o ar frio tende a se deslocar para baixo por ser mais denso (Çengel \& Boles, 2001) tornando a temperatura do layout das caixas inferior menor com valor médio de $23,0^{\circ} \mathrm{C}$. Entretanto, para a fisiologia do ovo fértil a temperatura dos dois layouts esteve acima do recomendado pela literatura, na qual é ressaltado que os ovos férteis devem 
ser armazenados em condição de temperatura ambiente abaixo $20{ }^{\circ} \mathrm{C}$, ou seja, abaixo do zero fisiológico (Fasenko et al., 1991; 1992). Quando essa temperatura se eleva o desenvolvimento embrionário é acelerado antes dos ovos serem incubados, o que resultará, como sequência, em perdas produtivas tais como a perda de água excessiva dos ovos, mortalidade e má-formação embrionária, diminuição da eclodibilidade e qualidade de pintos de corte (Reijrink et al., 2008; Rocha et al., 2008; Elibol \& Brake, 2008a e 2008b; Araújo et al., 2009; Reijrink et al., 2009; 2010; Nazareno, 2012).

Para a variável umidade relativa o maior valor médio foi encontrado no layout das caixas inferiores com $84 \%$, ocasionado pelas perdas de água dos ovos férteis durante o transporte e lavagem do caminhão na entrada do matrizeiro por questões de controle de sanidade. No entanto, os dois layouts das caixas (superiores e inferiores) apresentaram valores médios de umidade relativa acima de $75 \%$ de acordo com Araújo et al. (2009) pois se tem, com o aumento da umidade relativa, o início da condensação sobre os ovos que resultará em um ambiente mais propício para o crescimento de micro-organismos (fungos e bactérias) nos ovos férteis (Moura et al., 2008; Baracho et al., 2010; Freitas et al., 2011).

O maior valor médio de entalpia específica foi verificado no layout das caixas inferiores com $62,2 \mathrm{~kJ} \mathrm{~kg}^{-1}$ de ar seco, gerado pela elevação da temperatura e umidade relativa. Quanto maior o valor de entalpia específica maior também a quantidade de energia térmica existente no ar seco (baú do caminhão). Para determinada temperatura a elevação da umidade relativa resulta no aumento da entalpia específica.

Por meio dos resultados da análise geoestatística é possível observar que todas as variáveis ambientais para os dois layouts apresentaram dependência espacial ao longo do perfil térmico do caminhão (Tabela 2).

Tabela 2. Modelo e parâmetros estimados dos semivariogramas experimentais para as variáveis microclimáticas temperatura (T), umidade relativa (UR) e entalpia específica (h) em relação aos tratamentos de diferentes layouts das caixas superiores e inferiores no transporte de ovos férteis

\begin{tabular}{|c|c|c|c|}
\hline & $\begin{array}{c}\mathrm{T} \\
\left({ }^{\circ} \mathrm{C}\right)\end{array}$ & $\begin{array}{l}\text { UR } \\
\text { (\%) }\end{array}$ & $\begin{array}{c}\mathrm{h} \\
\left(\mathrm{kJ} \mathrm{kg}^{-1} \text { ar seco) }\right.\end{array}$ \\
\hline & \multicolumn{3}{|c|}{ Layout das caixas superiores do caminhão } \\
\hline$R^{2}$ & 0,31 & 0,70 & 0,76 \\
\hline $\mathrm{C}_{0}$ & 0,58 & 112,00 & 4,80 \\
\hline $\mathrm{C}_{0}+\mathrm{C}_{1}$ & 2,20 & 634,90 & 60,60 \\
\hline C & 1,62 & 522,09 & 55,80 \\
\hline IDE (\%) & 70,37 & 80,24 & 90,21 \\
\hline Alcance (m) & 3,45 & 6,49 & 3,73 \\
\hline \multirow[t]{2}{*}{ Modelo } & Esférico & Gausiano & Gausiano \\
\hline & \multicolumn{3}{|c|}{ Layout das caixas inferiores do caminhão } \\
\hline$R^{2}$ & 0,40 & 0,40 & 0,64 \\
\hline $\mathrm{C}_{0}$ & 1,03 & 84,50 & 4,11 \\
\hline $\mathrm{C}_{0}+\mathrm{C}_{1}$ & 4,14 & 369,90 & 29,21 \\
\hline C & 3,11 & 285,40 & 25,10 \\
\hline IDE (\%) & 75,20 & 77,20 & 85,90 \\
\hline Alcance (m) & 4,16 & 5,00 & 3,02 \\
\hline Modelo & Gausiano & Exponencial & Gausiano \\
\hline
\end{tabular}

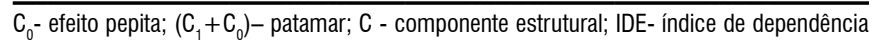
espacial
Para a umidade relativa os dados do layout das caixas superiores do caminhão foram ajustados ao modelo gaussiano com $\mathrm{R}^{2}$ e alcance de 0,70 e $6,49 \mathrm{~m}$, respectivamente e para entalpia específica, 0,76 e 3,73 m, respectivamente refletindo assim a continuidade da variável estudada. Essas também apresentaram forte dependência espacial com valores para umidade relativa e entalpia específica de 80,24 e 90,20\%, respectivamente em que, conforme Zimback (2001) o índice de forte dependência espacial (IDE) é $\geq 75 \%$. Apesar disto, a variável temperatura ajustou-se ao modelo esférico com $\mathrm{R}^{2}$ e alcance de 0,31 e 3,45 m, respectivamente, apresentando dependência espacial moderada, com valor de 70,34\% em que, segundo Zimback (2001) o índice de dependência espacial (IDE) nesta classificação se enquadra entre 25 e $75 \%$.

Os dados do layout das caixas inferiores do caminhão para temperatura se ajustaram ao modelo gaussiano com o $\mathrm{R}^{2}$ e alcance de 0,40 e 4,16 m, respectivamente, e para entalpia específica de 0,64 e 3,02 m, respectivamente. Porém a umidade relativa ajustou-se ao modelo exponencial com $\mathrm{R}^{2}$ e alcance de 0,40 e 5,00 m, respectivamente. Para este layout todas as variáveis estudadas ( $T$, UR e h) obtiveram forte dependência espacial com valores de 75,20, 77,20 e 85,90\%, respectivamente, de acordo com Zimback (2001).

Observa-se, por meio da temperatura, umidade relativa e entalpia específica, que os perfis microclimáticos do baú climatizado evidenciam um ambiente bastante heterogêneo para o transporte dos ovos férteis (Figura 2).

A.
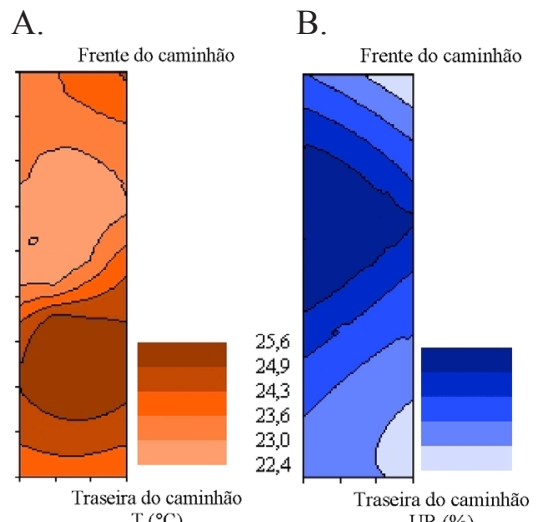

C.

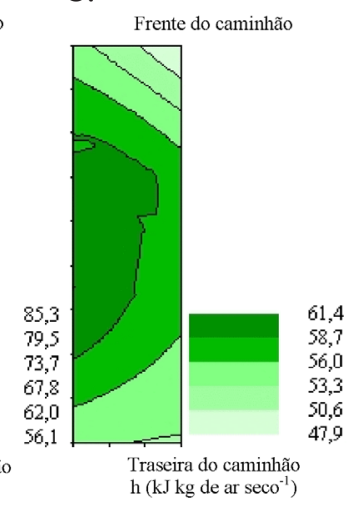

Figura 2. Distribuição espacial dos perfis térmicos da temperatura $(T)$, umidade relativa (UR) e entalpia específica (h) em relação ao layout das caixas superiores de ovos no transporte de ovos férteis

A temperatura do baú do caminhão em que os ovos férteis estavam inseridos, esteve acima do recomendado por Fasenko et al. (1991; 1992), Elibol \& Brake (2008a; 2008b) porém se observa grande heterogeneidade de temperatura ao longo do perfil do caminhão, cujas maiores temperaturas ocorrem basicamente na traseira do caminhão, de forma gradativa, com valores médios de 24,$3 ; 24,9$ e $25,6^{\circ} \mathrm{C}$.

A umidade relativa do baú do caminhão esteve acima do recomendado por Araújo et al. (2009) na maior parte do perfil do caminhão demonstrando forte heterogeneidade no perfil do caminhão com valores médios de 85,3; 79,5 e $73,7 \%$ respectivamente, localizados nas regiões próximas ao climatizador e centro do baú do caminhão. O excesso de 
umidade relativa pode estar relacionado também com as perdas de água dos ovos uma vez que é o local onde se tem uma quantidade maior de ovos, esperando-se que os ovos comecem a a perder água a partir da postura.

A amplitude do índice entalpia de conforto apresentou forte heterogeneidade no perfil térmico do caminhão com valores médios de 61,4; 58,7 e $56 \mathrm{~kJ} \mathrm{~kg}^{-1}$ de ar seco, localizados nas regiões próximas ao climatizador e centro do baú do caminhão. Como a entalpia é a relação entre a temperatura e umidade relativa pode-se dizer que os valores médios de entalpia do ambiente de transporte de ovos férteis tendem a se manter fora do recomendado, por conta da elevada variação das duas variáveis anteriores.

As regiões centro e traseira da carga do caminhão no layout das caixas superiores foram as de piores condições microclimáticas para os ovos férteis podendo caracterizar-se como possível causadora de perdas durante as operações préporteira no transporte de ovos férteis.

Resultados semelhantes obtiveram Damron et al. (1994), Gast et al. (2000), Anderson et al. (2008) e Nazareno (2012) que verificaram os maiores desvios de temperatura e umidade relativa perto das portas e próximo ao climatizador, no transporte de ovos comerciais.

É possível notar que as distribuições espaciais dos perfis microclimáticos do baú climatizado para as caixas inferiores de ovos obtiveram os menores valores médios de temperatura e entalpia específica em relação aos perfis térmicos do layout das caixas superiores de ovos (Figura 3).

A.

B.

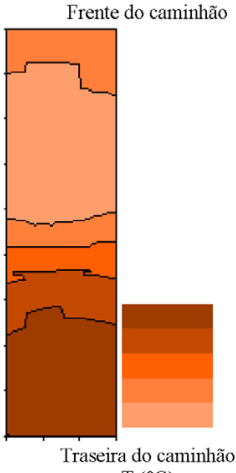

$\mathrm{T}\left({ }^{\circ} \mathrm{C}\right)$

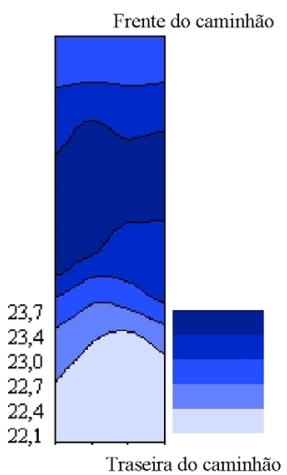

UR (\%)
C.

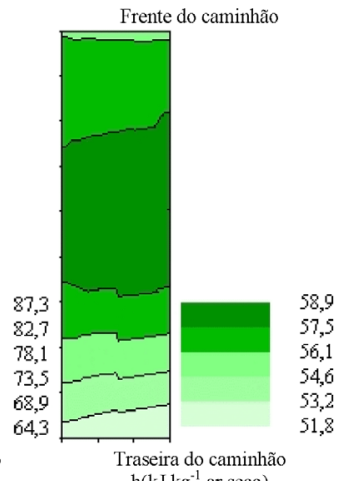

$\mathrm{h}\left(\mathrm{kJ} \mathrm{kg}^{-1}\right.$ ar seco)

Figura 3. Distribuição espacial dos perfis térmicos das variáveis temperatura do ar (T), umidade relativa (UR) e entalpia (h) em relação ao layout das últimas caixas de ovos no transporte de ovos férteis

Ao verificar a temperatura do ambiente do baú do caminhão nota-se que a mesma está acima do ideal, conforme Fasenko et al. (1991; 1992), Elibol \& Brake (2008a; 2008b); entretanto, é visível a forte heterogeneidade da temperatura ao longo do perfil do caminhão, com valores médios de 23,7; 23,4 e $23{ }^{\circ} \mathrm{C}$; também é possível observar que as maiores temperaturas ocorreram na região da traseira divergindo, assim, do layout das caixas superiores em que as regiões de maiores temperaturas ocorreram no meio e na traseira. Apesar das temperaturas dos dois layouts estarem acima do recomendado pela literatura, mesmo assim é possível verificar que o layout das caixas inferiores apresentou melhor condição

de conforto térmico para o transporte de ovos férteis nesta etapa pré-porteira, em relação ao layout das caixas de ovos superiores.

A distribuição espacial da umidade relativa no baú do caminhão encontra-se maior do que o recomendado por Araújo et al. (2009) apresentando forte heterogeneidade no perfil do caminhão com valores médios de 87,3; 82,7 e 78,1\%, localizados na região do centro do baú e na região próxima ao climatizador do caminhão. Comportamento semelhante ao da região de maiores umidades relativas foi encontrado no layout das primeiras caixas de ovos.

A distribuição espacial da variação entálpica no baú do caminhão obteve uma forte heterogeneidade no perfil térmico, com valores médios de 58,9; 57,5 e $56,1 \mathrm{~kJ} \mathrm{~kg}^{-1}$ de ar seco, localizados nas regiões do centro e próximo ao climatizador do baú do caminhão. Pelo fato da temperatura do baú do caminhão estar mais baixa no layout das caixas de ovos inferiores colaborando para que os valores de entalpia específica ficassem mais baixos, mesmo com a umidade relativa alta em relação ao layout das primeiras caixas de ovos, demonstrando que a temperatura do ambiente tende a ser inversamente proporcional à umidade relativa (Çengel \& Boles, 2001; Nazareno, 2012).

Assim, a região da traseira da carga no layout das caixas inferiores foi a mais problemática para a variável temperatura apresentando maiores valores mas as regiões do centro apresentaram o maior valor de umidade relativa e entalpia específica. Em diversas pesquisas com transporte de ovos comerciais foram encontrados resultados similares (Damron et al., 1994; Gast \& Holt, 2000; Anderson et al., 2008; OviedoRondón et al., 2009; Nazareno, 2012).

O layout das caixas superiores de ovos apresentou um perfil inadequado ao conforto térmico para o transporte de ovos férteis. É notável que as regiões de piores condições microclimática nos dois layouts se situam nas partes central e traseira, o que as coloca como regiões da carga mais suscetíveis à ocorrência de perdas pré-porteira durante o transporte dos ovos férteis.

Para reduzir as perdas pré-porteira é importante levar em consideração as questões de ambiência no transporte de ovos férteis; referida redução pode ser feita através da diminuição no número de caixas de ovos por pilhas, para se ter melhores condições de microclimáticas dentro do baú do caminhão e aumentar o número de viagens para o transporte da carga, o que poderá reduzir a desuniformidade térmica na carroceria do caminhão.

\section{Conclusões}

1. As condições microclimáticas no transporte de ovos férteis estiveram acima do zero fisiológico, podendo não garantir a paralisação do desenvolvimento embrionário dos ovos para os dois layouts das caixas de ovos.

2. O layout das caixas superiores registrou maiores valores de temperatura e entalpia, exceto para umidade relativa.

3. As regiões de piores condições microclimáticas nos dois layouts se situaram nas partes central e traseira o que torna essas regiões mais suscetíveis à ocorrência de perdas pré-porteira durante o transporte dos ovos férteis. 


\section{Literatura Citada}

Anderson, K. E.; Patterson, P. H.; Koelkebeck, K. W.; Darre, M. J.; Carey, J. B.; Ahn, D. U.; Ernst, R. A.; Kuney, D. R.; Jones, D. R. Temperature sequence of eggs from oviposition through distribution: Transportation - Part 3. Poultry Science, v.87, p.1195-1201, 2008.

ANUALPEC - Anuário da Pecuária Brasileira. São Paulo: FNP. 2011. 256p.

Araújo, W. A. G.; Alebrante, L.; Castro, A. D. Fatores capazes de afetar os indicies de eclosão. Revista Eletrônica Nutritime, v.6, p.1072-1087, 2009.

Baracho, M. S.; Nääs, I. A.; Gigli, A. C. S. impacto das variáveis ambientais em incubatório de estágio múltiplo de frangos de corte. Engenharia Agrícola. v.30,p.563-577, 2010.

Barbosa Filho, J. A. D.; Vieira, F. M. C.; Silva, I. J. O.; Garcia, D. B.; Silva, M. A. N.; Fonseca, B. H. F. Transporte de frangos: Caracterização do microclima na carga durante o inverno. Revista Brasileira de Zootecnia, v.38, p.2442-2446, 2009.

Çengel, Y. A.; Boles, M. A. Termodinâmica. 3.ed.Mcgraw-hill de Portugal, Alfragide, 2001. 900p.

Damron, B. L.; Douglas, C. R.; Jacobs, R. D. Temperature patterns in commercial egg transport vehicles. Journal of Applied Poultry Research, v.3, p.193-198, 1994.

Elibol, O.; Brake, J. Effect of egg position during three and fourteen days of storage and turning frequency during subsequent incubation on hatchability of broiler hatching eggs. Poultry Science, v.87, p.1237-1241, 2008a.

Elibol, O.; Brake, J. Effect of egg weight and position relative to incubator fan on broiler hatchability and chick quality. Poultry Science, v.87, p.1913-1918. 2008b.

Fasenko, G. M.; Hardin, R. T.; Robinson, F. E. Relationship of hen age and egg sequence position with fertility, hatchability, viability, and preincubation embryonic development in broiler breeders. Poultry Science, v.71, p.1374-1383, 1992.

Fasenko, G. M. P.; Robinson, F. E.; Armstrong, J. G.; Church, J. S.; Hardin, R. T. J. N. Variability in preincubation embryo development in domestic fowl: Effects of nest holding time and method of egg storage. Poultry Science, v.70, p.187-188, 1991.

Freitas, L. W.; Paz, I. C. L. A.; Garcia, R. G.; Caldara, F. R.; Seno, L. O.; Felix, G. A.; Lima, N. D. S.; Ferreira, V. M. O. S.; Cavichiolo, F. Aspectos qualitativos de ovos comerciais submetidos a diferentes condições de armazenamento. Revista Agrarian, v.4, p.66-72, 2011.

Gast, R. K.; Holt, P. S. Influence of the level and location of contamination on the multiplication of Salmonella enteritidis at different storage temperatures in experimentally inoculated eggs. Poultry Science, v.79, p.559-563, 2000.

Libardi, P. L.; Manfron, P. A.; Moraes, S. O.; Tuon, R. L. Variabilidade da umidade gravimétrica de um solo hidromórfico. Revista Brasileira de Ciência do Solo, v.20, p.1-12, 1996.

Moura, A. M. A.; Oliveira, N. T. E.; Thiebaut, J. T. L.; Melo, T. V. Efeito da temperatura de estocagem e do tipo de embalagem sobre a qualidade interna de ovos de codornas japonesas (Coturnix japonica). Ciência e Agrotecnologia, v.32, p.578-583, 2008.
Nazareno, A. C. Ambiência pré-porteira: avaliação das condições bioclimáticas e das operações pré-eclosão na qualidade de pintos de corte. Piracicaba: ESALQ/USP, 2012. 207p. Tese Doutorado

Oviedo-Rondón, E. O.; Wineland, M. J.; Small, J.; Cutchin, H.; Mcelroy, A.; Barri, A.; Martin, S. Effect of incubation temperatures and chick transportation conditions on bone development and leg health. Journal of Applied Poultry Research, v.18, p.671-678, 2009.

Reijrink , I. A. M.; Berghmans, D.; Meijerhof, R.; Kemp , B.; Brand, H. van den. The chicken embryo and its micro environment during egg storage and early incubation. World's Poultry Science, Journal, v.64, p.581-598, 2008.

Reijrink, I. A. M.; Berghmans, D.; Meijerhof, R.; Kemp, B.; van den Brand, H. Influence of egg storage time and preincubation warming profile on embryonic development, hatchability, and chick quality. Poultry Science, v.89, p.1225-1238, 2010.

Reijrink, I. A. M.; Meijerhof, R.; Kemp, B.; Graat, E. A. M.; Brand, H. van den. Influence of prestorage incubation on embryonic development, hatchability, and chick quality. Poultry Science, v.88, p.2649-2660, 2009.

Robertson, G. P. GS+: Geostatistics for the environmental sciences- GS+ User's Guide. Plainwell: Gamma Design Software, 2000. 152p.

Rocha, J. S. R.; Lara, L. J. C.; Baião, N. C.; Cançado, S. V.; Baião, L. E. C.; Silva, T. R. Efeito da classificação dos ovos sobre o rendimento de incubação e os pesos do pinto e do sacovitelino. Arquivo Brasileiro de Medicina Veterinária e Zootecnia, v.60, p.979-986, 2008.

Rodrigues, V. C.; Silva, I. J. O.; Vieira, F. M. C.; Nascimento, S. $\mathrm{T}$. A correct enthalpy relationship as thermal comfort index for livestock. International Journal of Biometeorology, v.55, p.455-459, 2011.

SAS - Statistic Alanalysis System: Realease 9.1.3, (software). Cary: SasInstitute, 2009. 620p.

Tanure, C. B. G. S.; Café, M. B.; Leandro, N. S. M.; Baião, N. C.; Tringhini. J. H.; Gomes, N. A. Efeitos da idade da matriz leve e do período de armazenamento de ovos incubáveis no rendimento de incubação. Arquivo Brasileiro de Medicina Veterinária e Zootecnia, v.61, p.1391-1396, 2009.

Trangmar, B. B.; Yost, R. S.; Uehara, G. Application sofgeo statistics to spatial studies of soil properties. Advances in Agronomy, v.38, p.45-94, 1985.

Vieira, S. R. Geoestatística em estudos de variabilidade espacial do solo. In: Novais, F.; Alvarez, V.; Schaefer, C. E. G. R. (ed.). Tópicos em ciência do solo. Viçosa: Sociedade Brasileira de Ciência do Solo, v.1, 2000. p.1-53.

Yassin, H.; Velthuis, A. G. J.; Boerjan, M.; Riel, J. van; Huirne, R. B. M. Field study on broiler eggs hatchability. Poultry Science, v.87, p.2408-2417, 2008.

Zimback, C. R. L. Análise espacial de atributos químicos de solos para fins de mapeamento da fertilidade. Botucatu: FCA/UNESP, 2001. 114p. Tese Livre-Docência 